\title{
The effect of three-dimensional cultured adipose tissue-derived mesenchymal stem cell-conditioned medium and the antiaging effect of cosmetic products containing the medium
}

\author{
Kyung Hye Kim ${ }^{1,2}$, Yun-Sik Kim ${ }^{3}$, Sunray Lee ${ }^{4^{*}}$ and Sungkwan $\mathrm{An}^{5^{*}}$
}

\begin{abstract}
Background: Recently, investigators have been trying to apply the by-products as well as stem cells themselves to various fields such as pharmaceuticals, medical devices, quasi-drug, cosmetis, etc. We aimed to comfirm the antisenescence effect of 3D cultured adipose tissue-derived mesenchymal stem cell-conditioned medium (3D cultured ADMSCs-CM) and develop them as cosmetic raw materials for anti-aging purposes.

Methods: We investigated the effect of 3D cultured ADMSCs-CM on collagen production and performed efficacy tests to evaluate the effect of a cream-based cosmetic product containing the medium using various methods, such as dermal density, skin moisture retention, and so on.

Results: Analysis of the effect of ADMSCs-CM on skin regeneration and production of collagen showed 1.5-fold (2D cultured ADMSCs-CM) and 2.5-fold (3D cultured ADMSCs-CM) increase in expressions of procollagen and 4-fold (2D cultured ADMSCs-CM) and 5-fold (3D cultured ADMSCs-CM) increase in the expression of collagen compared with control. In addition, related gene expression was also increased. We conducted a human skin test using a cream-based product containing 3D cultured ADMSCs-CM. In skin texture assessment, skin roughness decreased by $11.94 \%$ at the application site and $3.74 \%$ at the non-application site after 3 weeks of use. Compared with before cream use, after 2 and 4 weeks of substance use, the skin elasticity analysis showed an increase in the elasticity value by $5.97 \%$ and $9.34 \%$, respectively, and the improvement of small wrinkles was $5.01 \%$ and $6.23 \%$, respectively. After 2 and 4 weeks of test substance use, dermal density analysis showed $6.97 \%$ and $12.53 \%$ increase, respectively. Skin moisture retention analysis showed skin moisture maintained at $543.60 \%$ and $452.38 \%$, respectively, immediately after one-time use and after 20 min of cool breeze exposure compared with before application of the test substance.

Conclusions: As raw material for cosmetic products, 3D cultured ADMSCs-CM prevented skin aging by promoting collagen production, restoring damaged skin, and increasing dermal density. Therefore, 3D cultured ADMSCs-CM can be widely applied to maintain and improve skin condition.
\end{abstract}

Keywords: Stem cell-conditioned medium, Antiaging, Wrinkle, Moisturization, Collagen production

\footnotetext{
*Correspondence: sunray@naver.com; ansungkwan@konkuk.ac.kr

${ }^{4}$ Stem Cell Niche Division, CEFO Research Center, 45-13 Ujeongguk-ro, Jongno-gu, Seoul 03150, Republic of Korea

${ }^{5}$ Department of Cosmetics Engineering, Konkuk University, 120

Neungdong-ro, Gwangjin-gu, Seoul 05029, Republic of Korea

Full list of author information is available at the end of the article
}

(c) The Author(s). 2019 Open Access This article is distributed under the terms of the Creative Commons Attribution 4.0 International License (http://creativecommons.org/licenses/by/4.0/), which permits unrestricted use, distribution, and reproduction in any medium, provided you give appropriate credit to the original author(s) and the source, provide a link to the Creative Commons license, and indicate if changes were made. The Creative Commons Public Domain Dedication waiver (http://creativecommons.org/publicdomain/zero/1.0/) applies to the data made available in this article, unless otherwise stated. 


\section{Background}

Skin is the largest organ of the body, comprising the dermis and epidermis. The epidermis, the outermost skin layer, protects the skin from external stimuli and is constantly exposed to ultraviolet ray, heat, fine dust, and physical and chemical stimuli from the environment (Kim et al. 2019). Some of the external stimuli induce the production of reactive oxygen species, which cause epidermal cell damage and cell necrosis, resulting in acceleration of skin aging, such as wrinkles and loss of elasticity (Kim et al. 2011; Yoon et al. 2013).

Advances in modern medical science have increased life expectancy, resulting in growing interests in health and aging along with the higher expectation in the field of cosmetics. Pharmaceutical and biotechnology companies have focused on the development of highperformance products based on genes, proteins, and cells, using cutting-edge techniques such as stem cell culture and exosome extraction ( $\mathrm{Li}$ et al. 2019; Patel et al. 2019). The demand for high-performance cosmetics created a new field called cosmeceuticals, a hybrid field of cosmetics and pharmaceuticals, which is emerging as a global trend. For example, there has been remarkable progress in the development of cosmeceutical products with added pharmacological efficacies that slows down the loss of skin functions or regenerates damaged skin (Brandt et al. 2011).

In our study, we used a stem cell-conditioned medium as the main active ingredient for the development of a cosmetic product. Stem cells are broadly divided into adult stem cells and pluripotent stem cells. We used adult stem cells as they are relatively free from ethical issues and easier to culture compared with pluripotent stem cells. The safety of adult stem cells has been verified in many studies. Adult stem cells are frequently present in many adult tissues and have an excellent regenerative ability thanks to their multipotency (Weissman 2000; Weissman et al. 2001; Broxmeyer 2005). Adipose-derived mesenchymal stem cells (ADMSCs), a type of adult stem cells, are readily obtained from human tissues and easy to culture. For this reason, they have been used in the development of new drugs and for other various purposes. Adipose tissue is a good source of stem cells because it can be obtained in a large quantity and easily expanded in culture (Wilson et al. 2011; Strem et al. 2005; Zuk et al. 2001). Culture medium is an indispensable reagent in ADMSC culture and the depleted medium used to be discarded once the cell culture was finished. However, since the discovery of various growth factors in the used medium, the used medium was named as adipose-derived mesenchymal stem cell-conditioned medium (ADMSC-CM) and a substantial amount of efforts has been made to utilize it as an active ingredient for cosmetic products (Kim et al. 2007; Kim et al. 2009).

Wound healing is facilitated by the secreted factors such as growth factors from mesenchymal stem cells (MSCs) rather than the direct action of MSCs (Iso et al. 2007; $\mathrm{Fu}$ et al. 2007). The stem cell-conditioned medium contains various growth factors such as epidermal growth factor (EGF), vascular endothelial growth factor (VEGF), and collagen (Fu et al. 2007). They promote the expression of the genes involved in wound healing and fibroblast activity, thereby increasing collagen biosynthesis, resulting in reduction of wrinkles (Kim et al. 2009; Kim and Kim 2011), facilitation of wound healing in a wound model of nude rat (Nakagawa et al. 2005), and anti-oxidation effect by alleviating oxidative stresses on the skin (Kim and Kim 2011). Due to its property to promote skin regeneration, the conditioned media can be used as an active ingredient for cosmetic products. However, the present commercial media for stem cell culture contain substances such as phenol red and antibiotics, which are not approved for cosmetic products. Thus, it is useful to develop cell culture media made with the ingredients approved for cosmetics so that the conditioned media can be used in the development of high-performance cosmetic products (Iudicone et al. 2014; Choi et al. 2012).

Stem cell-conditioned medium as an active ingredient of cosmetic products contains various growth factors such as EGF and VEGF, which were secreted during the stem cell culture (Schinköthe et al. 2008). Aside from the growth factors found in ADMSC-CM, collagen, the major component of the extra cellular matrix, is known to have antiaging effects such as skin regeneration and elasticity improvement (Kim et al. 2009; Fu et al. 2007; Nakagawa et al. 2005). Presently, most cell cultures are done on a two-dimensional (2D) matrix such as the surface of a cell culture plate, on which cells attach and grow. However, this technique cannot reproduce in vivo environment sufficient enough to support physiological functions, morphology, and gene expression of the cells cultured. Methods of three-dimensional (3D) cell culture have been developed to culture cells in a condition similar to their native environment (Kenny et al. 2007; Breslin 2013). Cell culture matrix is made of porous polymers or gels. Selection of a matrix in where cells are embedded is important in 3D culture, and the research and development in cell culture matrix have steadily progressed (Sachlos and Czernuszka 2003; Hoqrebe et al. 2017).

ADMSC-CM derived from 3D culture has been favored as an innovative material for the development of biocosmetics (Kim et al. 2019). ADMSCs-CM have many antiaging effects including wrinkle improvement, skin regeneration, and wound healing (Park et al. 2008). 
Many biocosmetic products containing ADMSC-CM derived from $2 \mathrm{D}$ culture (2D cultured ADMSCs-CM ) have been developed and tested (Park et al. 2008). However, research on the safety and efficacy of ADMSC-CM from $3 \mathrm{D}$ culture (3D cultured ADMSCs-CM ) is scarce and systematic research is required.

The goal of this study was to investigate the effect of $3 \mathrm{D}$ cultured ADMSCs-CM on collagen production, and cosmetic efficacy of a cream-based product containing 3D cultured ADMSCs-CM on skin texture and elasticity, wrinkle, dermal density, and skin moisture retention. We observed the antiaging effect of a cream-based cosmetic product containing 3D cultured ADMSCs-CM and proposed the potential use of $3 \mathrm{D}$ cultured ADMSCs-CM as a new active ingredient for the development of cosmetic products.

\section{Methods}

A declaration for the study was approved by the IRB committee of the Korea Institute of Dermatological Sciences (approval number: 1-70005239-AB-N-01-201711HR-120-01) in according to the Helsinki Declaration.

\section{Cell lines and cell culture}

Human adipose tissue-derived mesenchymal stem cells (hADMSCs) were obtained with the consent of the donors. They were first washed with phosphate buffered saline (PBS) and extracted using a cell extraction kit, SmartX ${ }^{\circ}$ (DongKoo Bio \& Pharma Co., Ltd.), which allows easy extraction of hADMSCs from tissues. Cells were cultured with CEFOgro ${ }^{\text {Tm }}$ ADMSC (CEFO Co., Ltd).

Human dermal fibroblasts (hDFs) were obtained from males of 20 years old or under and cultured with CEFOgro $^{\mathrm{Tw}}$ hDF (CEFO Co., Korea) supplemented with $1 \%$ penicillin/streptomycin (Gibco, UK) $(100 \mathrm{IU} / 50 \mu \mathrm{g} / \mathrm{mL})$. Serial culture was performed 3 days after cell inoculation.

All cell cultures were done in a $37^{\circ} \mathrm{C}$ incubator with $5 \% \mathrm{CO}_{2}$ and relative humidity of $>95 \%$ unless indicated otherwise.

\section{Three-dimensional ADMSC culture}

Three-dimensional (3D) gel matrixes with a series of concentrations for every $5 \%$ from 5 to $30 \%$ were prepared with a biodegradable synthetic biogel (BASF, Germany). Powder of the biogel was melted in sterilized deionized water. Approximately $250 \mu \mathrm{L} / \mathrm{cm}^{2}$ of the melted gel was spread on a polymer membrane of 0.4$1 \mu \mathrm{m}$ thick (Corning, USA) and solidified at $37^{\circ} \mathrm{C}$ for 90 min to produce $3 \mathrm{D}$ cell culture matrix. Next, hADMSCs from 2D culture were inoculated on the biogel matrix and cultured in a $37^{\circ} \mathrm{C}$ incubator with $5 \% \mathrm{CO}_{2}$ and relative humidity of $>95 \%$. The medium was replaced 1 day after the cells stabilized.

\section{Preparation of ADMSC-conditioned medium}

A conditioned medium from 2D culture was produced by inoculating ADMSC at the cell density of 12,000 cells $/ \mathrm{cm}^{2}$ on a standard culture plate. Next day, the cells were washed with PBS three times and fresh CEFOgro $^{\text {mix }}$ XF ADMSC (CEFO Co., Korea) was added. The cells were cultured for one more day. The medium was collected and centrifuged at $1500 \mathrm{rpm}$ for $5 \mathrm{~min}$ to obtain the supernatant. The supernatant was filtered using a $0.22-\mu \mathrm{m}$ filter to obtain 2D cultured ADMSCs-CM.

To obtain 3D cultured ADMSCs-CM, ADMSC was inoculated at the density of 12,000 cells $/ \mathrm{cm}^{2}$. Next day, the cells were washed with PBS three times and CEFOgro $^{\text {Tn }} \mathrm{XF}$ ADMSC was added. The cells were cultured for one more day. The medium was harvested and centrifuged at $1500 \mathrm{rpm}$ for $5 \mathrm{~min}$ to obtain the supernatant. The supernatant was filtered with a $0.22-\mu \mathrm{m}$ filter to obtain a 3D cultured ADMSCs-CM.

\section{Protein quantitation of procollagen and collagen}

To assess the effects of the ADMSC-CM on the production of procollagen and collagen, human skin-derived fibroblasts were seeded into a 12-well plate (Corning, USA) at $2 \times 10^{4} / \mathrm{cm}^{2}$. The medium was replaced $24 \mathrm{~h}$ later. A negative control was cultured with a basal medium without serum, and a positive control was cultured in a basal medium supplemented with $0.04 \%$ adenosine. The experimental group was treated with stem cell-conditioned media derived from the $2 \mathrm{D}$ or $3 \mathrm{D}$ culture for $48 \mathrm{~h}$. ELISA Kit (Takara, Japan) and human Type 1 Collagen ELISA kit (R\&D systems, USA) were used to measure the protein amounts of procollagen and collagen. The experiment was conducted following the manual provided by the kit manufacturers.

\section{RT-qPCR for procollagen, collagen and lysyl hydroxylase}

To measure the gene expression of procollagen, collagen, and lysyl hydroxylase (LH), human skin-derived fibroblasts were seeded on a 6-well plate (Corning, USA) at $2 \times 10^{4} / \mathrm{cm}^{2}$, and the medium was replaced $24 \mathrm{~h}$ later. The negative control was cultured in a medium without serum. The positive control was cultured in a medium supplemented with $0.04 \%$ adenosine but no serum. The experimental group was grown in the stem cell-conditioned media from the 2D or 3D culture for $48 \mathrm{~h}$. The cells were washed with PBS twice on the culture plates, and mRNA was extracted using Trizol (Invitrogen, USA).

cDNAs were synthesized using a cDNA synthesis kit (ReverTra ACE qPCR RT Master, Toyobo Co.). PCR was performed for 35 cycles at $94{ }^{\circ} \mathrm{C}$ for $30 \mathrm{~s}, 58^{\circ} \mathrm{C}$ for $30 \mathrm{~s}$, and $72^{\circ} \mathrm{C}$ for $30 \mathrm{~s}$. The PCR products were resolved and detected in gel electrophoresis and visualized using LAS4000 (GE healthcare Co.). The PCR primers for 
procollagen were TGC CGT GAC CTC AAG ATG TGC C and CAT CCA CAA GCG TGC TGT AGG TG for the forward and reverse primers, respectively. For collagen primers, the forward primer was TGC CGA TGT CGC TAT CCA and the reverse was TCT TGC AGT GAT AGG TGA TGT TCT G. For LH primers, the forward primer was GGA ACC TGG CCT ATG ACA CCC $\mathrm{T}$ and the reverse primer was TGC CAT GCT GTG CCA GGA ACT. A set of $\beta$-actin primers was used as a control. These were ATC TGG CAC ACC TTC TAC AAT GAG CTG CG and CGT CAT ACT CCT GCT TGC TGA TCC ACA TCT GC for the forward and the reverse primers, respectively.

\section{Production of a cream-based product containing 3D cultured ADMSCs-CM}

A water phase was added in a main beaker, stirred and heated up to $80^{\circ} \mathrm{C}$. A liquid crystal emulsifier was then added to the heated water phase and hydrate at 6000 rpm using a homogenizer at $80^{\circ} \mathrm{C}$ for $10 \mathrm{~min}$. An oil phase in a separate container was heated up to $80^{\circ} \mathrm{C}$, added to the main beaker containing the water phase, and emulsified using a homogenizer at $6000 \mathrm{rpm}$ for 5 min at $75^{\circ} \mathrm{C}$. The mixture was cooled to $45^{\circ} \mathrm{C}$. A liquid crystal capsule phase was stirred and encapsulated in another container. The liquid capsule phase was then added to the mixture and mixed by homogenizer at $45^{\circ} \mathrm{C}$ and $6000 \mathrm{rpm}$ for $3 \mathrm{~min}$. It was cooled to $33^{\circ} \mathrm{C}$ and de-aerated. A colorless, opaque, cream-based substance containing 1\% 3D cultured ADMSCs-CM was prepared for the human skin test.

\section{Human skin test}

To assess the efficacy of cream-based cosmetic products containing 3D cultured ADMSCs-CM, it was performed with the approval of the Korea Institute of Dermatological Sciences.

\section{Subjects}

Volunteers were recruited for the human skin test of cream-based cosmetic products containing 3D cultured ADMSCs-CM. Female adults aged 30-60 years were selected. The participants were fully informed regarding the study and voluntarily signed the consent form.

\section{Test substance usage and dose}

The test substance was stored at room temperature (5$25^{\circ} \mathrm{C}$ ) away from the exposure of high temperature and direct light.

(1) Thirty microliters of the cream containing 3D cultured ADMSCs-CM was applied on the left lower forearm of the subjects $\left(3.0 \times 3.0 \mathrm{~cm}^{2}\right)$ by a same researcher using a disposable syringe (SUNG
SHIM MEDICAL CO., LTD, Korea). Absorption was induced using a latex finger coat.

(2) An equal amount of cream containing 3D cultured ADMSCs-CM was evenly applied on the left upper forearm after treated with sodium lauryl sulfate (SLS; BioShop, Canada) by the subjects every day during the 4-week research period. The cream was applied twice a day in the morning and evening after washing the face.

(3) The subjects applied an equal amount of cream containing 3D cultured ADMSCs-CM evenly on their face twice every day in the morning and evening after washing the face for 4 weeks.

(4) During the skin test period, the subjects were instructed not to use any functional cosmetic products that may affect the test results such as eye cream, skin whitening cream, antiaging cream, and body moisturizer. They were also restricted from using facial masks or massage.

\section{Primary skin irritation assessment}

The back of the subjects was washed with $70 \%$ ethanol and dried. An 8-mm diameter Finn Chamber (Smart Practice, USA) containing $20 \mu \mathrm{L}$ of the test substance was applied on the back of the subjects for $24 \mathrm{~h}$. Skin irritation was examined at $30 \mathrm{~min}, 24 \mathrm{~h}$, and $48 \mathrm{~h}$ after the patch removal and graded by a dermatologist based on the guideline of the International Contact Dermatitis Research Group (Table 1).

\section{Assessment of skin texture damaged by external stimuli}

A Finn Chamber containing SLS was applied on both forearms of the subjects to induce skin irritation. ANTERA 3D (Miravex, Ireland) was used to measure the effect of the test substances on the damaged skin. The entire measurements were performed by a single researcher who evaluated the sites of forearms treated with or without the test substances. The measurement was done at the same sties by overlapping the images obtained before the application of the test substances to ensure the accuracy of the measurement. The measurements were performed before using the test substance (after SLS application) and 3 weeks after the use of the test substances.

Table 1 Criteria for human skin patch test by International Contact Dermatitis Research Group

\begin{tabular}{lll}
\hline Symbol & Score & Result \\
\hline- & 0 & Negative \\
\pm & 0.5 & Doubtful or slight reaction and erythema \\
+ & 1 & Erythema + Induration \\
++ & 2 & Erythema + Induration + Vesicle \\
+++ & 3 & Erythema + Induration + Bullae \\
\hline
\end{tabular}




\section{Dermal torque assessment}

Dermal Torque Meter (Dermal Torque Meter DTM310, Dia-Storn Ltd., UK) was used to assess improvement in dermal elasticity. A single researcher fixed a probe on the right cheek of all subjects with an adhesive tape and applied a rotational force at a uniform angle and with a uniform force for $10 \mathrm{~s}$ to measure dermal elasticity. Measurement using a measuring device was performed before applying the test substance and after 2 and 4 weeks of use.

\section{Wrinkle improvement assessment}

ANTERA 3D was used to assess wrinkle improvement. A single researcher evaluated the creases around the right eye for all participants. For the sake of measurement reproducibility, images obtained prior to the use of the test substance were overlapped to obtain measurements from the same location. Device-assisted measurement was performed before applying the test substance and after 2 and 4 weeks of use.

\section{Dermal density assessment}

DUB-Skin Scanner (Taberna pro medicum, Luneburg, Germany), a device that produces high-resolution ultrasound images, was used to assess improvements in dermal density. Ultrasound gel was applied on the test site, and the probe of DUB-Skin Scanner was positioned perpendicular to the skin. A single researcher pressed on the skin $3 \mathrm{~cm}$ next to the outer corner of the left eye to measure dermal density for all subjects. Device-assisted measurement was performed before applying the test substance and after 2 and 4 weeks of use.

\section{Assessment of skin moisture retention}

Epsilon E100 (Biox Systems Ltd., UK) was used to assess skin moisture retention in an environment with artificial cold air. A single researcher applied an equal amount of the test substance on the lower left forearm and exposed it to a cool wind of $17^{\circ} \mathrm{C} \pm 2{ }^{\circ} \mathrm{C}$ from a misting fan in the natural wind mode at the distance of $50 \mathrm{~cm}$ for 20 min. Device-assisted measurement was performed before application of the test substance, immediately after the first application, and after $20 \mathrm{~min}$ of exposure to the artificial cool breeze.

\section{Statistical analysis}

All statistical analyses were performed using SPSS 17.0 for Windows (IBM, USA).

The subjects' responses to the questionnaire were analyzed using mean, standard deviation, frequency, and percentage. A paired $t$ test was used to identify any significant improvements in various parameters of the skin condition.

\section{Results}

Protein measurement of procollagen and collagen in human fibroblasts

The effect of 2D cultured ADMSC-CM and 3D cultured ADMSC-CM was compared by measuring the expression levels of procollagen and collagen in the culture condition of human dermal fibroblasts. Negative control was cultured in a basal medium without serum. The positive control was fibroblasts grown in a basal medium with $0.04 \%$ adenosine, and the other group of fibroblasts were treated for $48 \mathrm{~h}$ with ADMSC-CMs derived 2D cultured ADMSCs-CM or 3D cultured ADMSCs-CM (Fig. 1).

The levels of procollagen (Fig. 1a) and collagen (Fig. 1b) expression under each condition are shown in Fig. 1. Collagen is a marker for skin regeneration and rejuvenation. The expression level of procollagen increased 1.5-fold (2D cultured ADMSCs-CM ) and 2.5fold (3D cultured ADMSCs-CM ) compared with the negative control (Fig. 1a). The level of collagen expression was also increased 4-fold (2D cultured ADMSCs-CM ) and 5-fold (3D cultured ADMSCs$\mathrm{CM}$ ) with respect to the negative control (Fig. 1b). The fibroblasts showed higher levels of procollagen and collagen expressions in 3D cultured ADMSCs$\mathrm{CM}$ than in 2D cultured ADMSCs-CM, and the difference was statistically significant. Thus, this result suggests that stem cell-conditioned medium derived from 3D culture can facilitate the production of extra cellular matrix (ECM) such as collagen.

\section{mRNA expression of procollagen, collagen, and lysyl hydroxylase in human dermal fibroblasts}

For the negative control, human dermal fibroblasts were cultured with hDF medium without serum. The positive control was fibroblasts grown in a basal medium with $0.04 \%$ adenosine. In addition, cells were incubated in 2D cultured ADMSCs-CM or 3D cultured ADMSCs-CM for $48 \mathrm{~h}$ and then harvested. The mRNA levels of extra cellular matrix (ECM) protein such as procollagen and collagen were measured, and also confirmed the gene expression of LH, which is known to promote the synthesis of procollagen and collagen (Hausmann 1967) (Fig. 2).

The expression of procollagen in human dermal fibroblast was higher in both $2 \mathrm{D}$ and $3 \mathrm{D}$ cultured ADMSC-CMs than the positive control $(0.04 \%$ adenosine). Conversely, cells incubated with 3D cultured ADMSCs-CM showed a higher level of collagen expression than those incubated with 2D cultured ADMSCs-CM, but showed a similar level of collagen expression compared with the adenosine-treated positive control. The highest level of $\mathrm{LH}$ expression was observed in the human dermal fibroblast incubated 

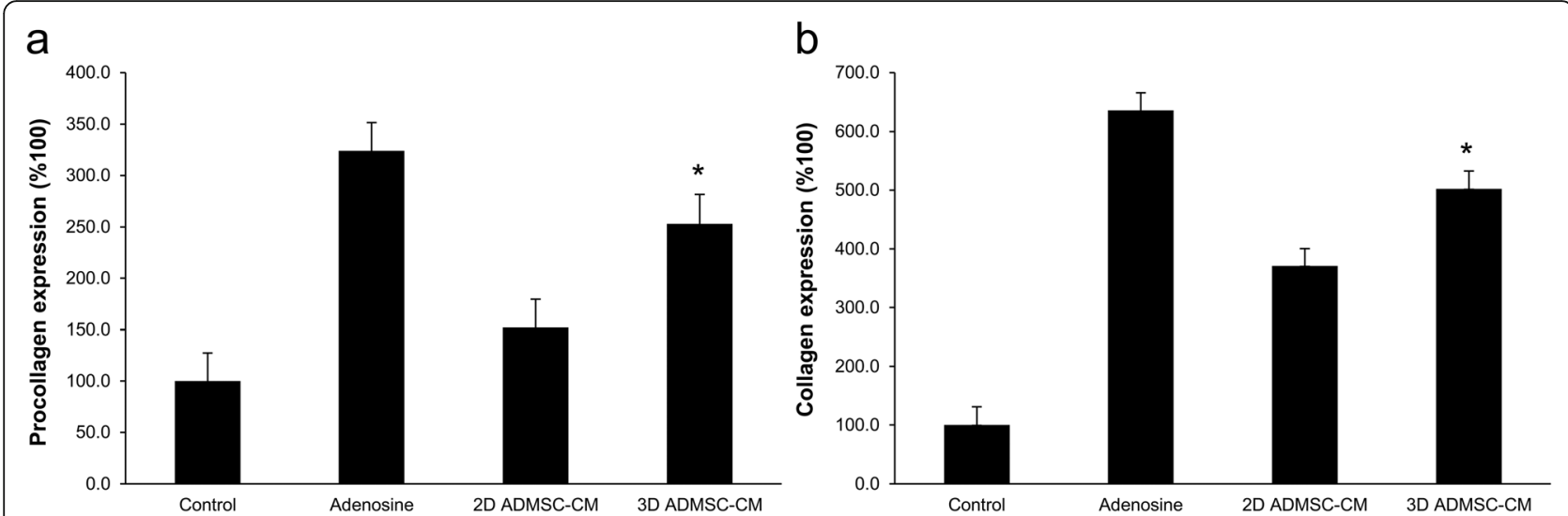

Fig. 1 Effects of 3D adipose-derived mesenchymal stem cell-conditioned medium on cell growth. a For the measurement of procollagen by ELISA, hDF $\left(2 \times 10^{4}\right.$ cells $\left./ \mathrm{cm}^{2}\right)$ were seeded in 12-well plates and treated with adenosine $0.04 \%, 2 \mathrm{D}$ cultured ADMSCs-CM, and 3D cultured ADMSCs-CM for $48 \mathrm{~h}$. Control is HDF culture medium without serum. $\mathbf{b}$ The expression of collagen was measured by preparing the cells in the same manner as for procollagen measurement. Data are expressed as mean $\pm \mathrm{SD}\left(n=3 ;{ }^{*} p<0.05\right)$

with 3D cultured ADMSCs-CM. The gene expression results of procollagen, collagen, and LH suggest that stem cell-conditioned medium promotes collagen production in human dermal fibroblast. Especially, 3D cultured ADMSCs-CM is expected to increase the production of ECM components in dermal cells.

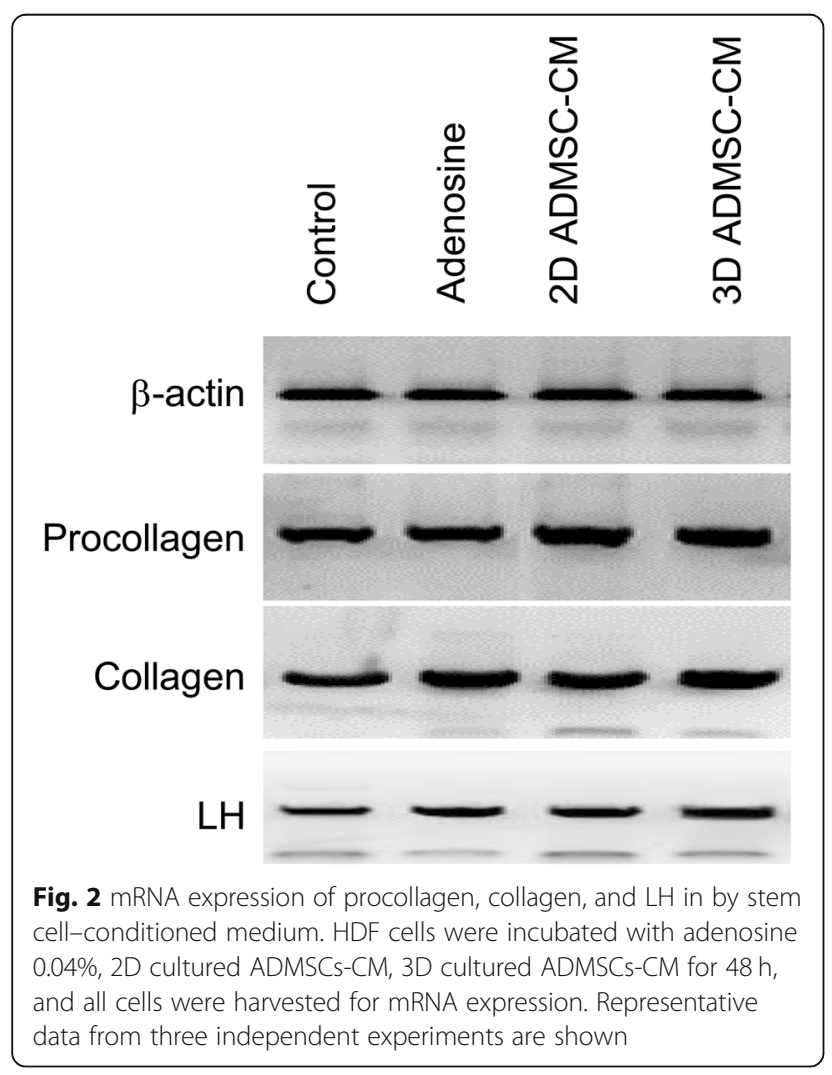

\section{Human skin test}

\section{Primary skin irritation test}

We conducted a primary skin irritation test using a cream-based cosmetic product containing 3D cultured ADMSCs-CM. Female volunteers in their twenties participated in the test.

After $0.5,24$, and $48 \mathrm{~h}$ following the removal of the patch, the dermatologist evaluated the degree of skin irritation based on the criteria provided by the International Contact Dermatitis Research Group (Table 1).

No signs of skin irritation such as erythema, swelling, and itching were observed at $30 \mathrm{~min}, 24 \mathrm{~h}$, and $48 \mathrm{~h}$ after the patch removal. Skin reactivity was 0.00 for all participants. Therefore, we conclude that the cream-based cosmetic product containing 3D cultured ADMSCs-CM does not induce skin irritation (Table 2).

\section{Efficacy assessment of cream-based cosmetic product containing $3 D$ cultured ADMSC-CM}

In this experiment, the efficacy of the cream-based cosmetic product containing 3D cultured ADMSCs-CM was assessed. The efficacy of the following criteria was assessed: skin texture restoration, dermal elasticity, wrinkle, dermal density, and skin moisture retention after exposure to an artificial cold breeze.

Table 2 Primary skin irritation study

\begin{tabular}{llllll}
\hline $\begin{array}{l}\text { Number of } \\
\text { participants }\end{array}$ & $\begin{array}{l}\text { Number of skin } \\
\text { irritation responders }\end{array}$ & \multicolumn{4}{l}{ Skin irritation degree } \\
\cline { 3 - 6 } & $30 \mathrm{~min}$ & $24 \mathrm{~h}$ & $48 \mathrm{~h}$ & Average \\
\hline 21 & 0 & 0.0 & 0.0 & 0.0 & 0.0 \\
\hline
\end{tabular}




\section{Assessment of improvement in skin texture damaged by external stimuli}

ANTERA 3D was used to evaluate the effects of the test substance on skin texture damaged by external stimuli. Figure 3 summarizes the assessments which were performed before and after 3-week treatment of the test substance.

In the analysis of skin texture damaged on both upper forearms using ANTERA 3D, the texture small values before (after SLS stimulation) and after the test substance use were compared. Texture small value is an indicator of skin roughness. The application sites of the test substance exhibited an $11.94 \%$ reduction of texture small value while the control sties showed a $3.74 \%$ reduction (Fig. 3a). The difference in skin texture was also observed in the images (Fig. 3b). On the basis of these results, we conclude that the cream-based cosmetic product containing 3D cultured ADMSCs-CM improves the texture of damaged skin more efficiently than the control product.

\section{Dermal elasticity assessment}

Dermal elasticity was examined using Dermal Torque Meter before and after 2- and 4-week treatments of the test substance (Fig. 4).

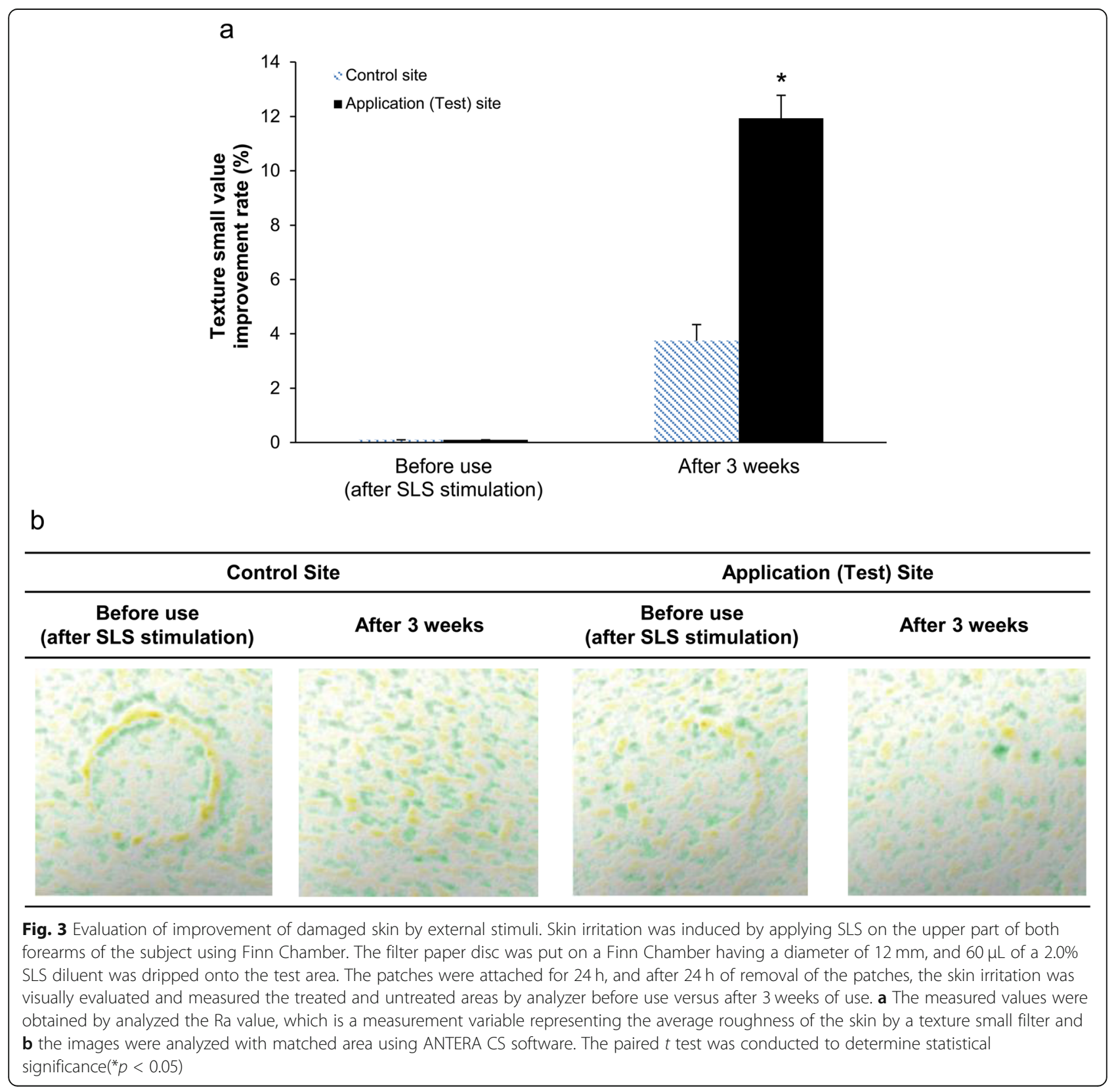




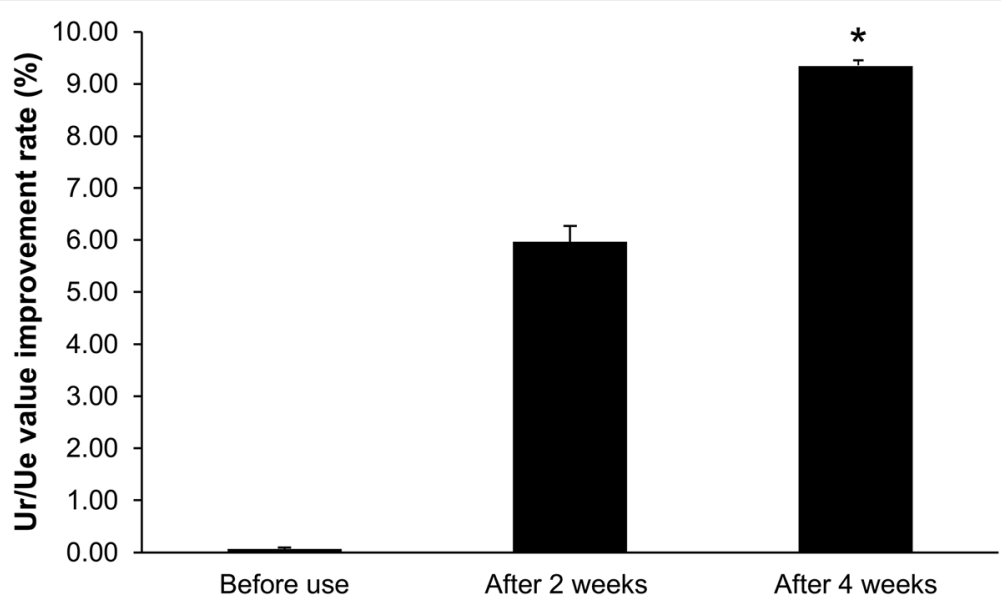

Fig. 4 Evaluation of deep skin elasticity evaluation in skin. To evaluate the improvement of the elasticity in deep skin by the test material, we fixed the probe of the Dermal Torque Meter to the right cheek of the face of the subject and measured the elasticity of deep skin by applying rotational force for $10 \mathrm{~s}$ at a constant angle and pressure at intervals of 2 weeks before and after use for up to 4 weeks. A ring with a 3-mm gap was attached to the Skin Torque Meter to transmit the rotational force to the dermal layer, and the Ur/Ue value indicating skin elasticity was analyzed. The paired $t$ test was conducted to determine statistical significance $\left(^{*} p<0.05\right)$

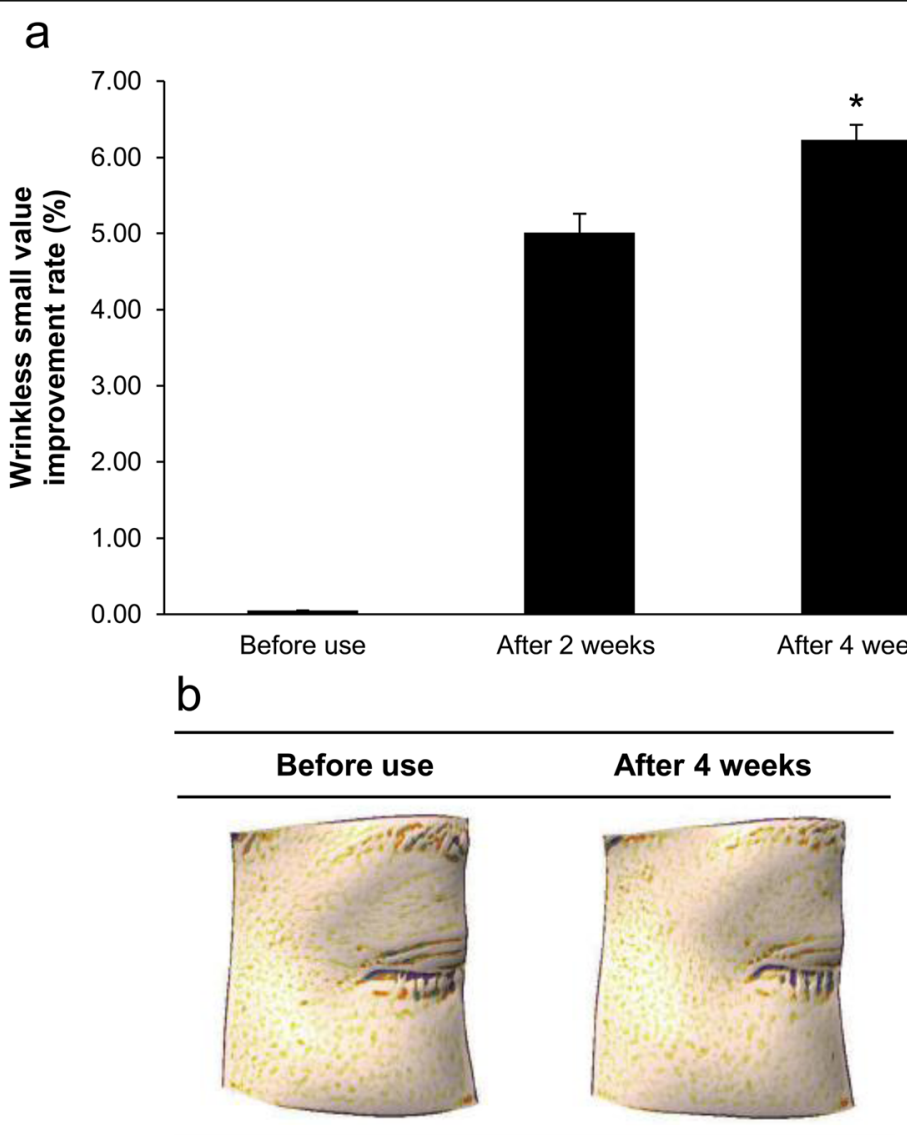

Fig. 5 Effect of anti-wrinkle. In order to effect of wrinkles care, the wrinkles of the right eye of all subjects were measured using ANTERA 3D and the same area was measured by overlapping with the images measured before use of the test substance for the reproducibility of the measurements. a The images were analyzed with matched area using ANTERA CS software and $\mathbf{b}$ wrinkles small value indicating the wrinkles of the skin was analyzed using indentation index at intervals of 2 weeks before and after use for up to 4 weeks. Paired $t$ test was conducted to determine statistical significance $\left.{ }^{*} p<0.05\right)$ 
To evaluate the improvement of the elasticity in deep skin by the test substance, we fixed the probe of Dermal Torque Meter to the right cheek of the face of the subject and measured the elasticity of deep skin by applying rotational force for $10 \mathrm{~s}$ at constant angle and pressure at intervals of 2 weeks before and after use for up to 4 weeks. A ring with a 3-mm gap was attached to the Skin Torque Meter to transmit the rotational force to the dermal layer and $\mathrm{Ur} / \mathrm{Ue}$ value indicating skin elasticity was analyzed. The paired $t$ test was conducted to determine statistical significance $(" p<0.05)$.

Figure 4 shows the assessment results of dermal elasticity using the Dermal Torque Meter before and after 2 and 4 weeks of the test substance use. The Ur/Ue value, an indicator of dermal elasticity, of the right cheek increased $5.97 \%$ and $9.34 \%$ after 2 and 4 weeks of the test substance use, respectively, compared with before use. This result suggests that the cream-based product containing 3D cultured ADMSCs-CM improves the dermal elasticity in deep skin.

\section{Wrinkle assessment}

Figure 5 shows the result of wrinkle assessments using ANTERA3D before and after 2 and 4 weeks of test substance use.

Wrinkles around the right eye crease improved by a $5.01 \%$ and $6.23 \%$ in the wrinkle small value, which measures wrinkle severity, following 2- and 4-week treatments of the test substance, respectively, compared with before use. These results demonstrate that the cosmetic product containing the 3D cultured ADMSCs-CM can improve wrinkles.

\section{Dermal density assessment}

The dermal density results obtained using DUB-Skin Scanner before use and 2 and 4 weeks after use of the test substance are shown in Fig. 6.

Analysis of the dermal density $3 \mathrm{~cm}$ from the outer corner of the left eye using DUB-Skin Scanner showed a $6.97 \%$ and $12.5 \%$ increase in dermal density after 2 and 4 weeks of use, respectively. These results indicate that

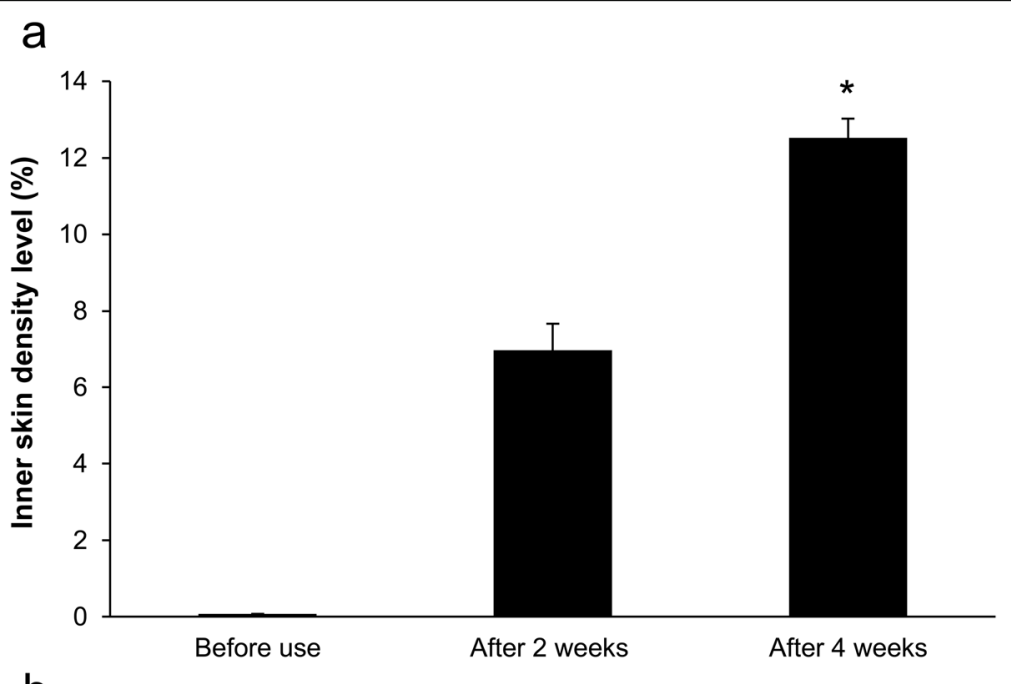

b

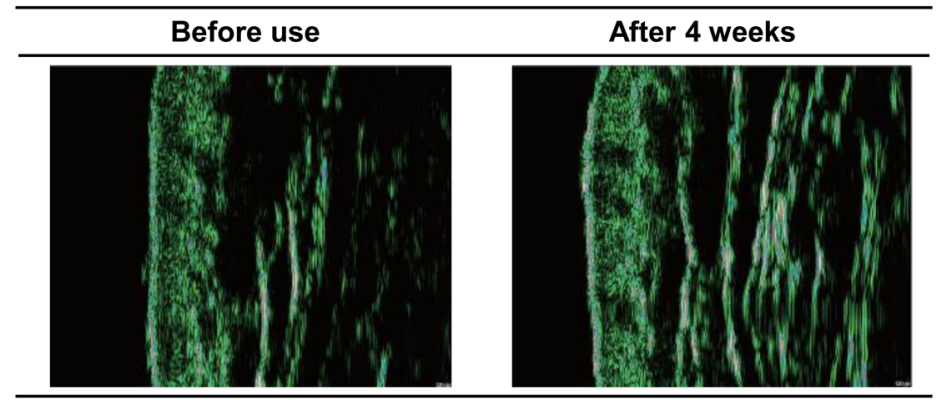

Fig. 6 Improvement of dermal density. To check of dermal density, the ultrasonic test gel was treated to the test site and the probe of the DUBSkin Scanner was perpendicular to the skin. Under the same conditions, the dermal density was measured by pressing all subjects at the same pressure on the $3-\mathrm{cm}$ area of the left eye tail. The range of analysis was limited from below the epidermis to the upper part of the subcutaneous fat, and the intensity indicating the density of the dermis was measured at intervals of 2 weeks before and after use for up to 4 weeks. a Graph is inner skin density level and $\mathbf{b}$ image shows the density of dermis layer. The paired $t$ test was conducted to determine statistical significance $\left({ }^{*} p\right.$ $<0.05)$ 
the cream-based cosmetic product containing 3D cultured ADMSCs-CM can improve dermal density.

\section{Assessment of skin moisture retention in an environment with artificial cool breeze}

Figure 7 shows the result of skin moisture retention analysis using Epsilon E100 before application of the test substance, immediately after application, and after 20 min of artificial cool breeze exposure.

Analysis of skin moisture retention in the left lower forearm was performed in an artificial wind environment. The result showed a $543.60 \%$ and $452.38 \%$ increases in skin moisture immediately after one-time application of the test substance and 20 min after cool breeze exposure, respectively, compared with before the application. The result suggests that the cream-based cosmetic product containing 3D cultured ADMSCs-CM enhances skin moisture retention in an environment similar to the artificial cool breeze.

\section{Discussion}

We arrived at the following conclusions based on the results of our experiments to verify the antiaging effect of 3D cultured ADMSCs-CM and its efficacy as an active ingredient in cosmetic product.

First, the effects of 2D and 3D cultured ADMSC-CMs on the production of collagen were evaluated against a known antiaging agent, adenosine, as a positive control. Collagen is a marker of skin regeneration and rejuvenation. We observed 1.5- and 2.5-fold increases of procollagen expression in the fibroblasts treated with $2 \mathrm{D}$ cultured ADMSC-CM and 3D cultured ADMSC-CM, respectively (Fig. 1a). The expression of collagen was also increased 4-fold with 2D cultured ADMSCs-CM and 5-fold with 3D cultured ADMSCs-CM, compared with the basal medium without serum, a negative control (Fig. 1b). The stem cell-conditioned media also increased the production of collagen, procollagen (a precursor of collagen), and lysyl hydrogenase in human epidermal keratinocytes. Especially 3D cultured ADMSCs-CM which was prepared by 3D culture

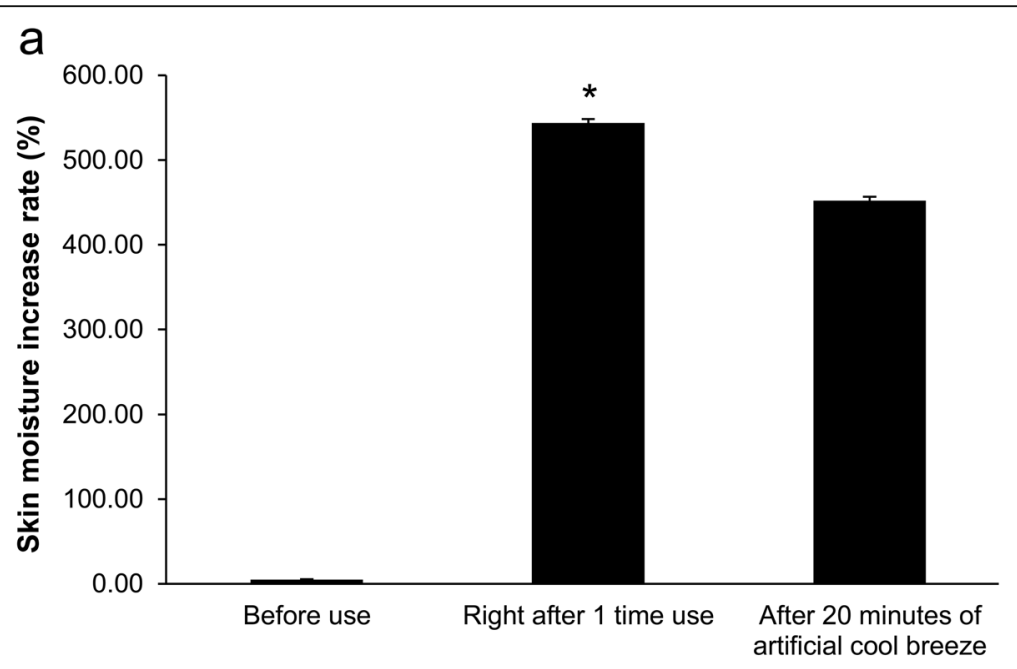

b

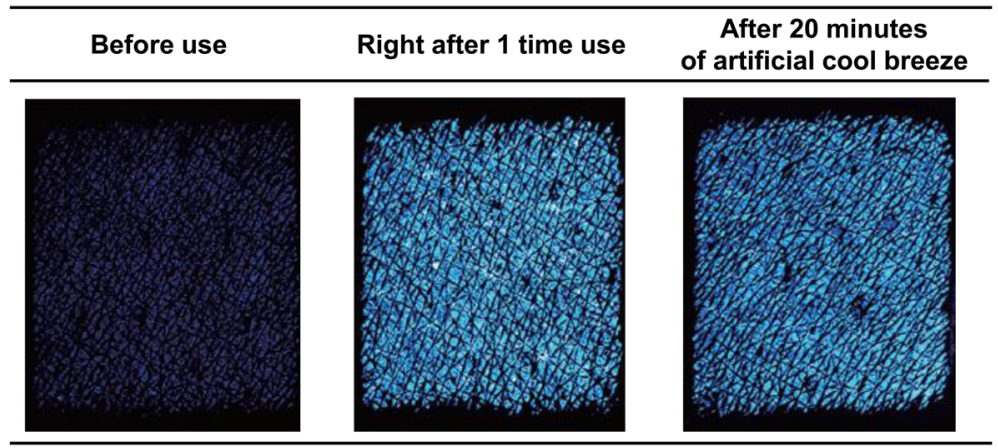

Fig. 7 Assessment of skin moisture retention effect in artificial cold wind environment. We applied the same amount of the test substance to the left lower forearm of the subject and analyzed the skin moisture increase rate at a temperature of $17^{\circ} \mathrm{C} \pm 2^{\circ} \mathrm{C}$ at 50 -cm distance in natural wind mode by cooler for 20 min to check the moisturizing effect. a Graph is skin moisture increase rate and $\mathbf{b}$ skin images after application of artificial cool breeze. The paired $t$ test was conducted to determine statistical significance $\left(^{*} p<0.05\right)$ 
significantly increased the expression of collagen, a component of ECM (Fig. 2). Reportedly, secretory factors produced by ADMSC have antiaging effects (Park et al. 2008; Son et al. 2015).

Second, the effect of ADMSC-CM on skin texture was examined with a SLS-induced skin damage model. The cream-based cosmetic product containing 3D cultured ADMSCs-CM exhibited three times greater improvement of the skin texture in the damaged skin than the control (Fig. 3).

Third, we also investigated the effect of ADMSC-CM on dermal elasticity (Fig. 4), wrinkle (Fig. 5), and dermal density (Fig. 6) as means to evaluate the antiaging effect and anti-wrinkle effect of stem cell-conditioned medium. We expect a cream-based cosmetic product containing 3D cultured ADMSCs-CM may prevent skin aging by reducing wrinkles and increasing dermal elasticity.

Fourth, the cream-based cosmetic product based on 3D cultured ADMSCs-CM showed excellent skin moisturizing effect (Fig. 7). Skin moisturization is essential to prevent skin damage due to external stimuli such as environmental changes and internal causes such as allergy and atopic dermatitis. As dry skin becomes an important issue, skin moisturizer products have been actively developed. However, the research on skin moisture retention is relatively lacking. We believe that $3 \mathrm{D}$ cultured ADMSCs-CM prevents water evaporation from the skin and improves moisture retention by increasing dermal elasticity, facilitating the recovery of damaged skin, and increasing the production of ECM components such as collagen. 3D cultured ADMSCs-CM as an active ingredient of a cosmetic product also exerted its antiaging effects by promoting collagen production, restoring the damaged skin, and increasing dermal density.

\section{Conclusions}

3D cultured ADMSCs-CM increased the expression of collagen, procollagen, and $\mathrm{LH}$ in human dermal fibroblasts. We expect that the increased production of ECM improves the skin texture of damaged skin due to external stimuli and reduces wrinkles by enhancing dermal elasticity, dermal density, and moisture retention. These effects of 3D cultured ADMSCs-CM were examined in the experiments with a cosmetic product containing 3D cultured ADMSCs$\mathrm{CM}$. In addition, further studies to identify elements that impart antiaging properties to 3D cultured ADMSCs-CM will broaden the application to related fields. In conclusion, the cream-based cosmetics containing 3D cultured ADMSCs-CM have antiaging properties and can be widely applied to improve skin condition.

\section{Abbreviations}

ADMSC: Adipose tissue-derived mesenchymal stem cells; BASF: Biodegradable synthetic biogel; ECM: Extra cellular matrix; EGF: Epidermal growth factor; hDF: Human dermal fibroblast; LH: Lysyl hydroxylase; PBS: Phosphate buffered saline; SLS: Sodium lauryl sulfate; VEGF: Vascular endothelial growth factor

\section{Acknowledgements}

Not applicable.

\section{Authors' contributions}

SL and SA designed the study. YSK formulated the cream. KHK and SL performed research, including the experiments, data collection, and statistical analysis. KHK, SL, and SA wrote the draft of the manuscript. All authors read and approved the final manuscript.

\section{Funding}

Not applicable.

\section{Availability of data and materials}

Not applicable.

\section{Ethics approval and consent to participate}

The subjects were informed about all the information with regard to the test; they participated after signing a written consent according to their will. All clinical tests for this study have undergone the deliberation of the Institutional Review

Boards before starting the tests (approval number: 1-70005239-AB-N-01201711-HR-120-01).

\section{Consent for publication}

Not applicable.

\section{Competing interests}

The authors declare that they have no competing interests.

\section{Author details}

'Department of Cosmetology, Graduate School of Engineering, Konkuk University, 120 Neungdong-ro, Gwangjin, Seoul 05029, Republic of Korea. ${ }^{2}$ New Business Team, DongKoo Bio \& Pharma, B-14F, 114, Beobwon-ro, Songpa-gu, Seoul 05854, Republic of Korea. ${ }^{3}$ R\&D Center, DongKoo Bio \& Pharma, 5F, 21, Daewangpangyo-ro 644 beon-gil, Bundang-gu, Seongnam-si, Gyeonggi-do 13494, Republic of Korea. ${ }^{4}$ Stem Cell Niche Division, CEFO Research Center, 45-13 Ujeongguk-ro, Jongno-gu, Seoul 03150, Republic of Korea. ${ }^{5}$ Department of Cosmetics Engineering, Konkuk University, 120 Neungdong-ro, Gwangjin-gu, Seoul 05029, Republic of Korea.

Received: 27 June 2019 Accepted: 14 November 2019

Published online: 31 December 2019

\section{References}

Brandt FS, Cazzaniga A, Hann M. Cosmeceuticals: current trends and market analysis. Semin Cutan Med Surg. 2011;30:141-3.

Breslin S. O' Driscoll L. Three-dimensional cell culture: the missing link in drug discovery. Drug Discov Today. 2013;18:240-9.

Broxmeyer HE. Biology of cord blood cells and future prospects for enhanced clinical benefit. Cytotherapy. 2005;7:209-18.

Choi EJ, Cho HB, Yoon HS. Developments of culture media for human skin stem cell, and evaluation of efficacy of cosmetics containing culture media. Asian J Beauty Cosmetol. 2012;10:949-60.

Fu X, Fang L, Li H, Li X, Cheng B, Sheng Z. Adipose tissue extract enhances skin wound healing. Wound Rep Reg. 2007;15:540-8.

Hausmann E. Cofactor requirments for the enzymatic hydroxylation of lysine in a polypeptide precursor of collagen. Biochem Biophys Acta. 1967;133:591-3.

Hogrebe NJ, Reinhardt JW, Gooch KJ. Biomaterial microarchitecture : a potent regulator of individual cell behavior and multicellular organization. J Biomed Mater Res A. 2017;105:640-61.

Iso Y, Spees JL, Serrano C, Bakondi B, Pochampally R, Song YH, et al. Multipotent human stromal cells improve cardiac function after myocardial infarction in mice without long-term engraftment. Biochem Biophys Res Commun. 2007; 354:700-6.

ludicone P, Fioravanti D, Bonanno G, Miceli M, Lavorino C, Totta P, et al. Phathogen-free, plasma-poor platelet lysate and expansion of human mesenchymal stem cells. J Transl Med. 2014;12:28. 
Kenny PA, Lee GY, Myers CA, Neve RM, Semeiks JR, Spellman PT, et al. The morphologies of breast cancer cell lines in three-dimensional assays correlate with their profiles of gene expression. Mol Oncol. 2007;1:84-96.

Kim J, Lee CW, Kim EK, Lee SJ, Park NH, Kim HS, et al. Inhibition effect of Gynura procumbens extract on UV-B-induced matrix-metalloproteinase expression in human dermal fibroblasts. J Ethnopharmacol. 2011;137:427-33.

Kim KH, Lee SR, Park HS. Inhibitory effects of three dimensional adipose tissuederived mesenchymal stem cell conditioned medium on immune response and efficacy evaluation of its cream. Asian J Beauty Cosmetol. 2019;17:25-36.

Kim SM, Kim BH. Anti-wrinkle effect of ADMSCs-CM in photoaging skin model of hairless mice. J Kor Soc Cosm. 2011;17:689-96.

Kim WS, Park BS, Sung JH. Protective role of adipose-derived stem cells and their soluble factors in photoaging. Arch Dermatol Res. 2009;301:329-36.

Kim WS, Park BS, Sung JH, Yang JM, Park SB, Kwak SJ, et al. Wound healing effect of adipose-derived stem cells: a critical role of secretory factors on human dermal fibroblasts. J Dermatol Sci. 2007;48:15-24.

Li Z, Hu C, Jia J, Xia Y, Xie H, Shen M, et al. Establishment and evaluation of a simple size-selective method for exosome enrichment and purification. J Biomed Nanotech. 2019;15:1090-6.

Nakagawa H, Akita S, Fukui M, Fujii T, Akino K. Human mesenchymal stem cells successfully improve skin-substitute wound healing. $\mathrm{Br} J$ Dermatol. 2005:153:29-36.

Park BS, Jang KA, Sung JH, Park JS, Kwon YH, et al. Adipose-derived stem cells and their secretory factors as a promising therapy for skin aging. Dermatol Surg. 2008;34:1323-6.

Patel GK, Khan MA, Zubair H, Srivastava SK, Khushman M, Singh S, et al. Comparative analysis of exosome isolation method using culture supernatant for optimum yield, purity and downstream applications. Sci Rep. 2019;9:5335.

Sachlos E, Czernuszka JT. Making tissue engineering scaffolds work. Review: the application of solid freeform fabrication technology to the production of tissue engineering scaffolds. Eur Cell Mater. 2003;5:29-40.

Schinköthe T, Bloch W, Schmidt A. In vitro secreting profile of human mesenchymal stem cells. Stem Cells Dev. 2008;17:199-206.

Son WC, Yun JW, Kim BH. Adipose-derived mesenchymal stem cells reduce MMP-1 expression in UV-irradiated human dermal fibroblasts : therapeutic potential in skin wrinkling. Biosci Biotechnol Biochem. 2015;79:919-25.

Strem BM, Hicok KC, Zhu M, Wulur I, Alfonso Z, Schreiber RE, et al. Multipotential differentiation of adipose tissue-derived stem cells. Keio J Med. 2005;54:132-41.

Weissman IL. Stem cells: units of development, units of regeneration, and units in evolution. Cell. 2000;100:157-68.

Weissman IL, Anderson DJ, Gage F. Stem and progenitor cells: origins, phenotypes, lineage commitments, and transdifferentiations. Annu Rev Cell Dev Biol. 2001;17:387-403.

Wilson A, Butler PE, Seifalian AM. Adipose-derived stem cells for clinic applications: a review. Cell Prolif. 2011;44:86-98.

Yoon YM, Bae SH, An SK, Choe YB, Ahn KJ, An IS. Effects of ultraviolet radiation on the skin and skin cell signaling pathways. Asian J Beauty Cosmetol. 2013; 11:417-26.

Zuk PA, Zhu M, Mizuno H, Huang J, Futrell JW, Katz AJ, et al. Multilineage cells from human adipose tissue: implications for cell-based therapies. Tissue Eng. $2001 ; 7: 211-28$

\section{Publisher's Note}

Springer Nature remains neutral with regard to jurisdictional claims in published maps and institutional affiliations.

Ready to submit your research? Choose BMC and benefit from:

- fast, convenient online submission

- thorough peer review by experienced researchers in your field

- rapid publication on acceptance

- support for research data, including large and complex data types

- gold Open Access which fosters wider collaboration and increased citations

- maximum visibility for your research: over $100 \mathrm{M}$ website views per year

At BMC, research is always in progress.

Learn more biomedcentral.com/submissions 